\title{
Subband Adaptive Array for DS-CDMA Mobile Radio
}

\author{
Xuan Nam Tran \\ Department of Electronic Engineering, The University of Electro-Communications, Chofugaoka 1-5-1, \\ Chofu-shi, Tokyo 182-8585, Japan \\ Email:namtx@radio3.ee.uec.ac.jp \\ Takanori Omata \\ Department of Electronic Engineering, The University of Electro-Communications, Chofugaoka 1-5-1, \\ Chofu-shi, Tokyo 182-8585, Japan \\ Core Network Planning Department and Switching Network Planning Department, Vodafone K.K., \\ Atago 2-5-1, Minato-ku, Tokyo 105-6205, Japan \\ Email: takanori.omata@vodafone.com

\section{Tetsuki Taniguchi} \\ Department of Electronic Engineering, The University of Electro-Communications, Chofugaoka 1-5-1, \\ Chofu-shi, Tokyo 182-8585, Japan \\ Email:taniguch@ee.uec.ac.jp
}

\section{Yoshio Karasawa}

Department of Electronic Engineering, The University of Electro-Communications, Chofugaoka 1-5-1, Chofu-shi, Tokyo 182-8585, Japan

Email: karasawa@ee.uec.ac.jp

\section{Received 15 April 2003; Revised 24 September 2003; Recommended for Publication by Mukund Padmanabhan}

We propose a novel scheme of subband adaptive array (SBAA) for direct-sequence code division multiple access (DS-CDMA). The scheme exploits the spreading code and pilot signal as the reference signal to estimate the propagation channel. Moreover, instead of combining the array outputs at each output tap using a synthesis filter and then despreading them, we despread directly the array outputs at each output tap by the desired user's code to save the synthesis filter. Although its configuration is far different from that of 2D RAKEs, the proposed scheme exhibits relatively equivalent performance of 2D RAKEs while having less computation load due to utilising adaptive signal processing in subbands. Simulation programs are carried out to explore the performance of the scheme and compare its performance with that of the standard 2D RAKE.

Keywords and phrases: subband adaptive array, CDMA, RAKE, multipath fading.

\section{INTRODUCTION}

Digital mobile communications are affected by multipath fading and interference causing reduced channel capacity and impaired signal quality. One approach to overcome the problem is to use the spread spectrum or specifically code division multiple access (CDMA). The use of orthogonal codes with large processing gain can help to reduce the cochannel interference (CCI) and prevent users from interfering with each other, that is, reduce the multiple access interference (MAI) [1]. Another approach to cancelling interference and increasing channel capacity is to employ array antenna at the base station. The use of an array antenna with an appropriate adaptive algorithm adds another dimension, namely, spatial dimension to channel estimation resulting in spatiotemporal signal processing which has been realised as an efficient scheme for improvement of capacity and interference suppression [2].

The combination of an array antenna and CDMA to maximise performance benefits was first presented by Compton in [3] and studied further in $[4,5,6,7,8,9]$. It was clearly shown that this combination helps to greatly reduce interference and improve channel capacity. When the RAKE receiver is integrated with an adaptive array to become a two-dimensional (2D) RAKE, multipaths are better combined since information from both spatial and temporal domains can be exploited to estimate the propagation channel. Several schemes of 2D RAKE have been proposed and 


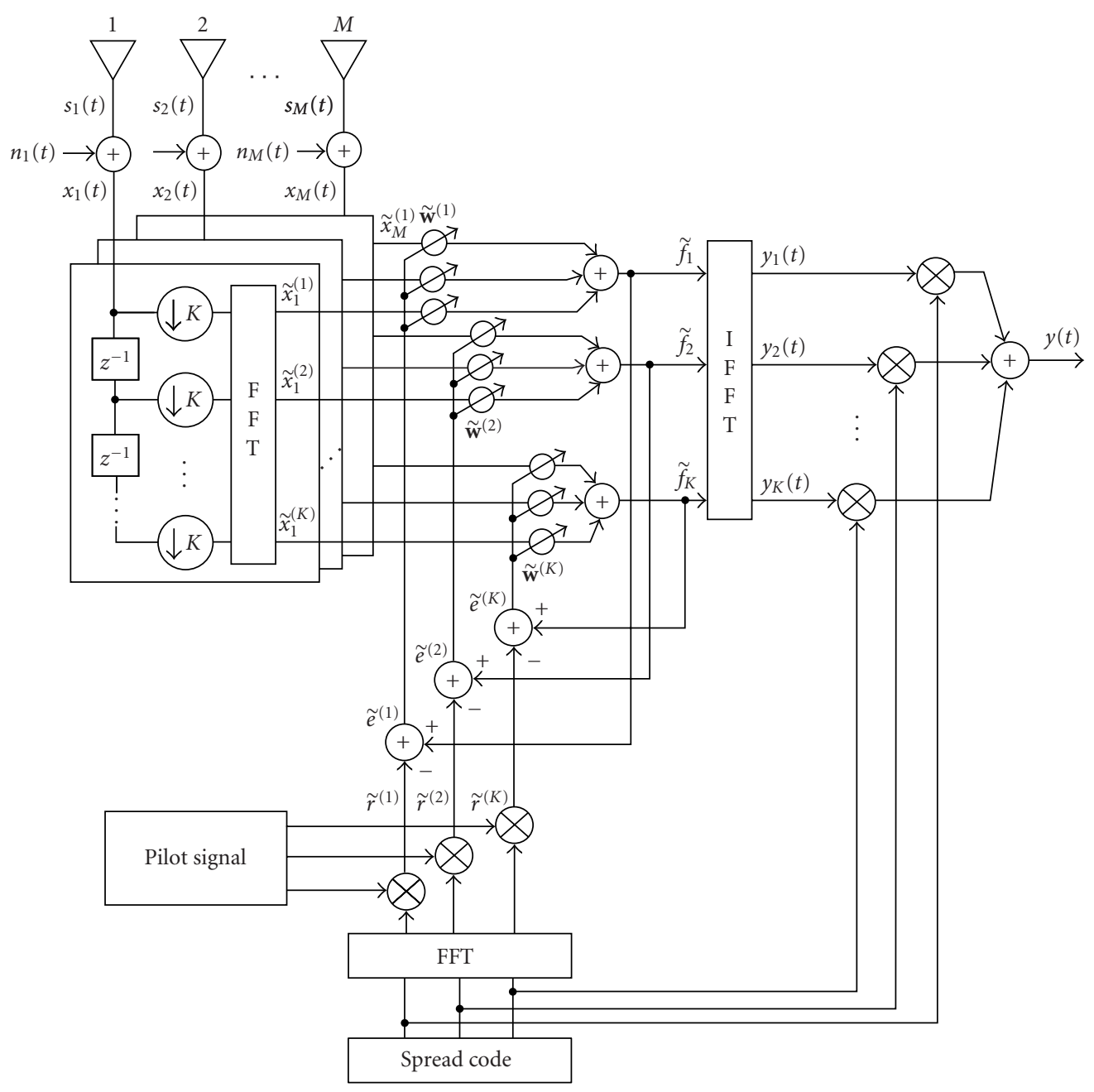

Figure 1: Subband adaptive array for DS-CDMA.

studied in $[5,6,7]$. A typical configuration of 2D RAKE receivers often contains a beamforming structure followed by a conventional one-dimensional (1D) RAKE as presented in $[6,7]$. By using these 2D RAKEs, the system performance has been shown to be greatly improved compared with that of 1D RAKE receiver alone or CDMA with adaptive array antenna without implementing RAKE combination. However, the beamforming structures used in these 2D RAKEs require large computation load which results in increased processing delay. The solution to reduce computational load is to use subband signal processing for array antenna or subband adaptive antenna (SBAA) which has been recently introduced in $[10,11,12]$.

SBAA utilises analysis filter bank to decompose the received signal into subbands and performs adaptive signal processing in each subband. The output signals at output taps are then reconstructed using synthesis filter bank. By doing so, the computational load of SBAA decreases significantly compared with broadband beamformers such as tapped delay line adaptive array (TDLAA) [13]. Moreover, compared with conventional adaptive arrays perform- ing only spatial processing (narrow-band beamformers), the use of SBAA helps to increase the correlation between multipaths [11] and allows implementation of parallel processing. Therefore, SBAA can be considered a prospective candidate for spatial-temporal processing.

In this paper, we propose a novel scheme of adaptive array for direct-sequence CDMA (DS-CDMA) using subband signal processing to make use of CDMA and SBAA advantages. The subband structure of the scheme is similar with that introduced in [10]. However, the method used to generate the reference signal and combine the array output used in the scheme is different. In our approach, we use the spreading code and pilot signal as the reference signal to estimate the propagation channel. To generate the reference signal, the user's spreading code is first transformed to the frequency domain using fast Fourier transform (FFT) and then this transformed code is used to despread the pilot signal. Moreover, instead of combining the array outputs at output taps using a synthesis filter and then despreading them, we despread directly the array outputs by the desired user's code and thus the synthesis filter is saved. Although 
its configuration is far different from that of the 2D RAKE receivers, the proposed scheme exhibits relatively equivalent performance while having less computation load due to utilising adaptive signal processing in subbands. For this reason, we call the scheme an implicit $2 D$ RAKE receiver.

We organise the rest of the paper as follows. In Section 2, we present the description of the proposed scheme of SBAA for CDMA, focusing on its capability to resolve multipath fading and suppress interference. In Section 3, we compare the performance of the implicit 2D RAKE with that of the standard 2D RAKE using simulation results obtained from computer programs. Finally, we conclude our paper in Section 4.

\section{SBAA FOR DS-CDMA}

\subsection{Configuration description}

In this section, we provide the description of the proposed SBAA scheme for DS-CDMA. The configuration of the scheme is shown in Figure 1.

Consider an asynchronous direct-sequence spread BPSK system, where after demodulation to remove the carrier frequency, the received signal of the $i$ th user is given by

$$
s_{i}(t)=\alpha_{i} c_{i}(t) b_{i}(t)
$$

where $\alpha_{i}$ is the complex amplitude of the received signal, $b_{i}(t)$ is the $i$ th user's symbol given for BPSK modulation as

$$
b_{i}(t)=b_{u} \in\{-1,1\}, \quad u T_{b} \leq t<(u+1) T_{b},
$$

and $c_{i}(t)$ is the spreading code assigned to the $i$ th user with

$$
c_{i}(t)=c_{v} \in\{-1,1\}, \quad v T_{c} \leq t<(v+1) T_{c} .
$$

In (2) and (3), $T_{b}$ and $T_{c}$ are the bit and chip intervals, respectively. In the practical systems, $T_{b}$ is often selected to be much larger than $T_{c}$ to have high processing gain, that is, $P_{G}=T_{b} / T_{c} \gg 1$.

Assume that the system is affected by multipath fading, where the received signal from the $i$ th user contains $P_{i}$ multipaths with different amplitudes $\alpha_{i, p}$, delays $\tau_{i, p}$, and arrival angles $\theta_{i, p}$. Taking into consideration the effect of all $I$ users and local noise, the received signal at the array can be written as

$$
\mathbf{x}(t)=\sum_{i=0}^{I-1} \sum_{p=0}^{P_{i}-1} \alpha_{i, p} b_{i}\left(t-\tau_{i, p}\right) c_{i}\left(t-\tau_{i, p}\right) \mathbf{a}\left(\theta_{i, p}\right)+\mathbf{n}(t)
$$

where $\mathbf{a}\left(\theta_{i, p}\right)$ is the array response vector corresponding to the $p$ th path of the $i$ th user's signal, and

$$
\mathbf{n}(t)=\left[\begin{array}{llll}
n_{1}(t) & n_{2}(t) & \cdots & n_{M}(t)
\end{array}\right]^{\mathrm{T}}
$$

is the noise vector containing independent and identically distributed (i.i.d) noise in each element. For a linear uniformly spaced array, $\mathbf{a}\left(\theta_{i, p}\right)$ is given by

$$
\mathbf{a}\left(\theta_{i, p}\right)=\left[\begin{array}{llll}
1 & e^{-j(2 \pi d / \lambda) \sin \theta_{i, p}} & \cdots & e^{-j(2(M-1) \pi d / \lambda) \sin \theta_{i, p}}
\end{array}\right]^{\mathrm{T}},
$$

where $\lambda$ is the signal wavelength, $d$ is the distance between array elements, and $[\cdot]^{\mathrm{T}}$ denotes the vector transpose operation.

Now if we define

$$
\mathbf{s}_{i, p}(t)=\alpha_{i, p} b_{i}\left(t-\tau_{i, p}\right) c_{i}\left(t-\tau_{i, p}\right) \mathbf{a}\left(\theta_{i, p}\right)
$$

as the signal vector received from the $p$ th path of the $i$ th user, then (4) can be rewritten as

$$
\mathbf{x}(t)=\sum_{i=0}^{I-1} \sum_{p=0}^{P_{i}-1} \mathbf{s}_{i, p}(t)+\mathbf{n}(t) .
$$

Next, the received signal $\mathbf{x}(t)$ is decimated with a decimation rate $D$ which is smaller than or equal to the number of subbands $K$ before being converted into frequency domain subband samples. For $D=K$, we have a critical sampling SBAA, and for $D<K$, we have an oversampling SBAA. In our approach, we use the critical sampling to reduce the complexity in generating the reference signal for the training process. As a result, the decimation rate $D$ in Figure 1 is equal to the number of subbands $K$. Since critical sampling is assumed, the analysis filter works as a serial-to-parallel (S/P) converter and converts serial signal samples into parallel subband samples. These subband samples in time domain are then transformed into frequency domain subband samples using (FFT). Denote bold symbols with an overhead tilde as vectors containing samples in the frequency domain, the subband signal vectors at $n$th subband in frequency domain are given by

$$
\widetilde{\mathbf{x}}^{(n)}=\sum_{i=0}^{I-1} \sum_{p=0}^{P_{i}-1} \widetilde{\mathbf{s}}_{i, p}^{(n)}+\widetilde{\mathbf{n}}^{(n)} .
$$

In order to perform the adaptive signal processing in subbands, it is necessary that the reference signal also be converted into frequency domain subbands as the received signal. In our proposed configuration of SBAA for DS-CDMA, the reference signal is generated from the desired user spreading code and the pilot signal. First, the user spreading code is transformed into frequency domain using the FFT transform, and then this frequency domain spreading code is used to spread the pilot signal. The result of this process is the frequency domain reference samples for each subband. Suppose that the 0th user is taken as the user of interest (desired user), while the rest $(I-1)$ users are uninterested (undesired users). Assume that the pilot signal of the 0 th user is $d_{0}(t)$, then the frequency domain reference samples at the $n$th subband are given by

$$
\tilde{r}^{(n)}=\sum_{k=1}^{K} c_{0}\left(t-[k-1] T_{c}\right) d_{0}(t) e^{-j(2 \pi / K)(n-1)(k-1)} .
$$

It should be pointed out that the spreading code length is equal to the number of subbands $K$, and thus the array 


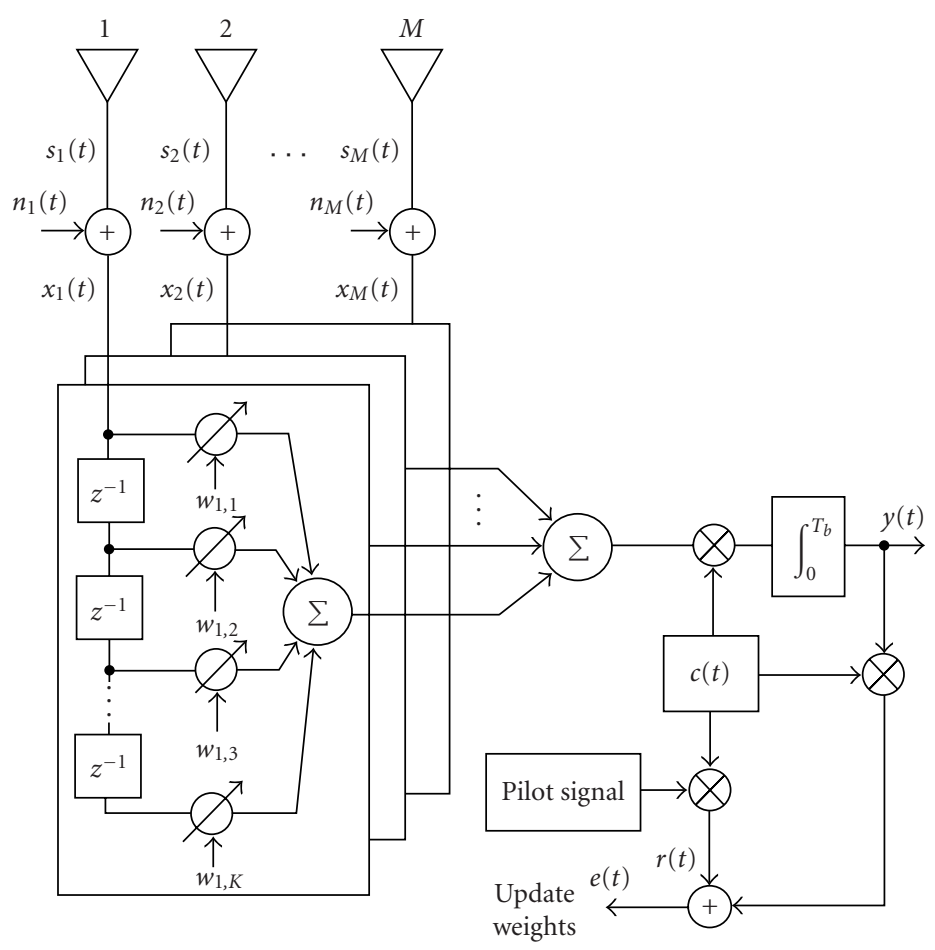

Figure 2: Standard 2D RAKE receiver for DS-CDMA.

configuration is dependent on the initial selection of the spreading code length.

In the training process, the complex weights in subbands are updated by the error signal defined as the difference between the combined subband signal $\tilde{f}^{(n)}$ and the reference signal in subbands $\tilde{r}^{(n)}$ as follows:

$$
\tilde{e}^{(n)}=\tilde{f}^{(n)}-\tilde{r}^{(n)}
$$

Using the mean square error $E\left[\left(\tilde{e}^{(n)}\right)^{2}\right]$ as a criterion to optimise the complex weights will result in the optimal weight vectors in subbands given by the well-known Wiener-Hopf equation

$$
\widetilde{\mathbf{W}}^{(n)}=\left(\widetilde{\mathbf{R}}^{(n)}\right)^{-1} \widetilde{\mathbf{p}}^{(n)},
$$

where $\widetilde{\mathbf{R}}^{(n)}=\mathrm{E}\left[\widetilde{\mathbf{x}}^{(n)}\left(\widetilde{\mathbf{x}}^{(n)}\right)^{\mathrm{H}}\right]$ are the covariance matrices and $\widetilde{\mathbf{p}}^{(n)}=\mathrm{E}\left[\widetilde{\mathbf{x}}^{(n)}\left(\tilde{r}^{(n)}\right)^{*}\right]$ are the reference correlation vectors in subbands. Here $\mathrm{E}[\cdot],(\cdot)^{*}$, and $(\cdot)^{\mathrm{H}}$ denote the expectation, the complex conjugate, and the Hermitian operation, respectively.

The subband signals after being weighted by the optimal weights are combined according to each subband and the inverse FFT (IFFT) is then performed on the subband combined signals $\tilde{f}^{(n)}$ to give the array outputs $y_{k}(t)$ in time domain. To convert these array outputs to the serial signal, a synthesis filter or a parallel-to-serial $(\mathrm{P} / \mathrm{S})$ converter for the case of the critical sampling SBAA is often needed $[12,13,14,15,16]$. Since the signal-to-interference-plus- noise ratio (SINR) performance of SBAA does not depend on the synthesis filter [12], in our approach instead of converting $y_{k}(t)$ into serial signal $y(t)$ and then despreading this serial signal, we despread directly $y_{k}(t)$ by the desired user's spreading code $c_{0}(t)$ to save the synthesis filter bank. The role of this despreading part is the same as that of the correlator in the direct-sequence spread BPSK receivers.

By using our proposed configuration of SBAA for DSCDMA, several advantages can be achieved including a $2 \mathrm{D}$ RAKE's function although its configuration is far different from that of the conventional 2D RAKE receiver. For this reason, hereafter we will call the proposed SBAA for DS-CDMA an implicit $2 D$ RAKE receiver.

\subsection{Implicit 2D RAKE versus 2D RAKE}

In this section, we compare the performance of the proposed implicit 2D RAKE with that of the standard 2D RAKE. A standard RAKE receiver often employs a TDL with complex weights to coherently/incoherently combine delayed paths to maximise the output SINR [8]. This standard RAKE is also referred to as $1 \mathrm{D}$ RAKE since only the temporal structure of the received signal is exploited to estimate the channel response [6]. Due to the increasing research results on spatiotemporal processing, a new configuration of RAKE which is called the spatio-temporal RAKE receiver has been introduced in $[5,6,7]$. The spatio-temporal RAKE, which is also known as 2D RAKE receiver, is an extension of 1D RAKE where a conventional time domain RAKE receiver is combined with an adaptive array antenna to exploit both spatial and temporal structures of the received signal for maximum 
power combination of delayed paths. Due to the additional spatial dimension, both multipath fading and MAI are better mitigated, leading to the increased channel capacity and improved output SINR [6]. When constructing 2D RAKE receivers for CDMA, there exist different methods to integrate 1D RAKE with an adaptive array antenna resulting in different variations of 2D RAKE such as those in $[5,6]$. In this paper, for the purpose of comparing our proposed implicit 2D RAKE with 2D RAKEs, we will consider only the standard 2D RAKE given in Figure 2. This standard 2D RAKE is similar to the one introduced in [7]. The main principle of the standard 2D RAKE is that the received signal at the $m$ th antenna $s_{m}(t)$ is first put through a TDL of length $K$. The output signals from the TDL are then multiplied with an optimum weight vector $\mathbf{w}_{m}=\left[\begin{array}{llll}w_{m, 1} & w_{m, 2} & \cdots & w_{m, K}\end{array}\right]^{\mathrm{T}}$ and combined together. After that, the combined signals from each antenna will be combined with each other and despread to give the output signal $y(t)$. Note that the received signals $s_{m}(t)$ are processed on the chip-by-chip basis by the standard 2D RAKE rather than the block-by-block mode as in the implicit 2D RAKE. Moreover, the process used to update weights in the standard 2D RAKE is done in time domain in contrast to that in the frequency domain in the implicit 2D RAKE.

Now we consider the implicit 2D RAKE presented in Section 2.1. The implicit 2D RAKE receiver is different from the 2D RAKE receivers presented so far $[5,6,7]$ in that it performs adaptive signal processing (beamforming) in subband frequency domain rather than in full-band time domain. By using subband frequency domain processing, the implicit 2D RAKE has the following performance characteristics compared with the standard 2D RAKE.

(i) Relatively equivalent performance. Since SBAA using FFT is a theoretically equivalent form of TDLAA, the performances of both the adaptive arrays are relatively equal. In [13], Compton has shown that the output SINR of TDLAA is identical to that of SBAA using FFT provided that the number of taps in TDLs is the same with the number of samples used by FFT. Consequently, the performance of the implicit 2D RAKE receiver is also the same as that of the standard 2D RAKE if the number of subbands $K$ of the implicit 2D RAKE is the same as the number of employed taps in the standard 2D RAKE. This is true for single path environment since the output SINRs of both the 2D RAKEs are given as a function of the number of antennas $M$, the processing gain $P_{G}$, and the input signal-to-noise ratio $\mathrm{SNR}_{\text {in }}$ as follows:

$$
\begin{aligned}
\operatorname{SINR}_{\text {out }}[\mathrm{dB}]= & 10 \log _{10}(M)+10 \log _{10}\left(P_{G}\right) \\
& +\operatorname{SNR}_{\text {in }}[\mathrm{dB}] .
\end{aligned}
$$

However, in multipath fading environment, the conclusion of [13] is no longer valid due to lack of considering the correlation between multipaths. Although subband signal processing has been shown to have capability to enhance the multipath correlation $[11,12]$, the performance of SBAA is in effect still not as good as that of TDLAA [12]. Assume that there are two multipaths with equal powers, incident at the array: the direct path with angle of arrival (AOA) of $0^{\circ}$ and the delayed path with $\mathrm{AOA}=30^{\circ}$. For a linear halfwavelength spaced array antenna, the two paths are orthogonal and thus totally uncorrelated. In this case, if the delay of the delayed path is smaller than the number of employed taps, the output SINR of the standard 2D RAKE is given by

$$
\begin{aligned}
\operatorname{SINR}_{\text {out }}[\mathrm{dB}]= & 10 \log _{10}(M)+10 \log _{10}\left(P_{G}\right) \\
& +10 \log _{10}(2)+\mathrm{SNR}_{\text {in }}[\mathrm{dB}],
\end{aligned}
$$

whereas the output SINR of the implicit RAKE decreases from the value of (14) to the value of (13) depending on the delay of the delayed path. When the delay is very large, there may be difference up to $3 \mathrm{~dB}$ in the output SINR of the two 2 RAKE receivers. The performance degradation of the implicit 2D RAKE can be attributed to the block mode, that is, decimation, in processing the received signals. As we will explain in the next part, by decimating the received signals, the 2D RAKE can achieve significantly reduced computational complexity sacrificing the multipath correlation. However, as clearly shown in [11], if the number of subbands, or equivalently the length of spreading code, is chosen large enough, the 2D RAKE can obtain almost full multipath correlation leading to smaller degradation. Moreover, it is noted that practical DSCDMA systems often suffer multipaths with delay of about several chips. Therefore, if the channel suffers from small delay and the length of spreading code is chosen large enough, the output SINR of the implicit 2D RAKE will be relatively equal to that of the standard 2D RAKE receiver. This conclusion will be supported by simulation results in Section 3 .

(ii) Reduced computational load. This can be seen by comparing the processing methods of the implicit and standard 2D RAKE receivers. While the standard 2D RAKE processes the received signal in the chip-by-chip basis, this is done on block-by-block mode by the implicit 2D RAKE. As a result, the implicit 2D RAKE requires less mathematical operations than the standard 2D RAKE does. For a $K$ tap and $M$ element array antenna, the standard 2D RAKE employing the sample matrix inversion (SMI) algorithm requires $(K M)^{3}$ multiplications for each weight update. The implicit 2D RAKE with $K$ subbands, on the other hand, needs only $K M^{3}$ multiplications [13]. Taking into account $2 K \log _{2} K$ multiplications due to both FFT and IFFT processing, the computational load required by the implicit RAKE is $K\left(M^{3}+2 \log _{2} K\right)$. Since DS-CDMA systems are often implemented with large processing gain $P_{G}$, then $K$ is large, and thus $(K M)^{3} \gg K\left(M^{3}+\right.$ $\left.2 \log _{2} K\right)$. Consequently, the use of the implicit $2 \mathrm{D}$ RAKE helps to save a considerably large amount of computational load. 


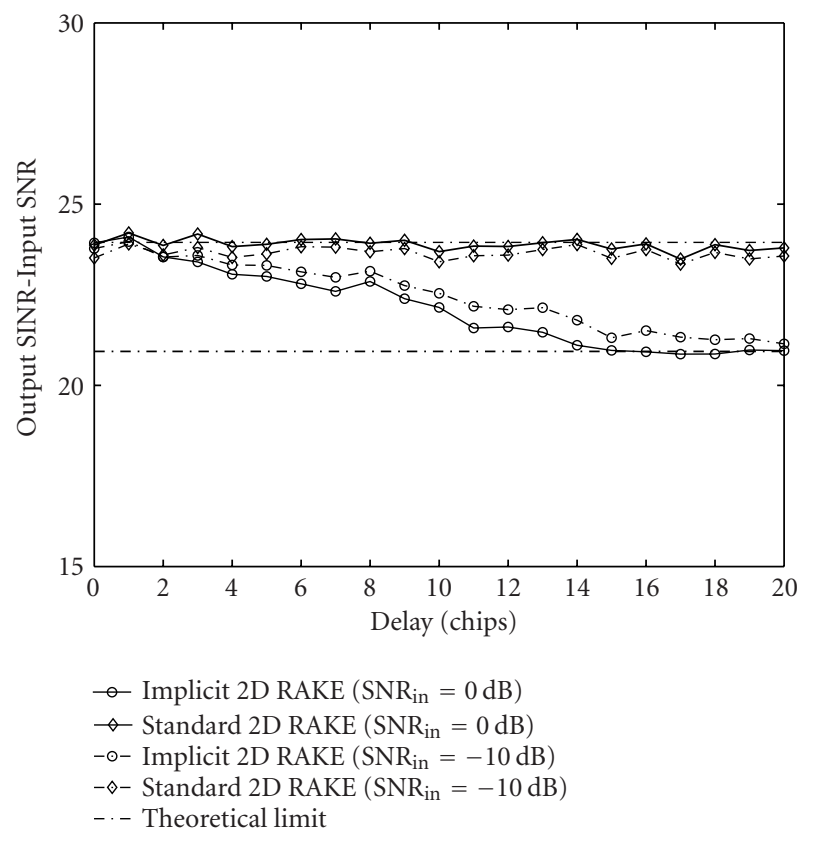

FIGURE 3: SINR versus delay of delayed path $\left(\theta_{0,0}=0^{\circ} / \tau_{0,0}=0\right.$ chip, $\theta_{0,1}=30^{\circ}$ ).

(iii) Parallel structure. Parallel structure is an advantage of the implicit 2D RAKE over the standard 2D RAKE. The parallel structure of the implicit 2D RAKE allows implementation of parallel processing, that is, distribution of tasks over different digital signal processors (DSPs), which is very convenient for constructing high complexity systems such as an adaptive array with a large number of antenna elements.

\section{SIMULATION RESULTS}

In this section, we carry out the performance analysis of the implicit 2D RAKE using simulation results by computer programs. We will focus our analysis mainly on two capabilities of the implicit 2D RAKE: (i) multipath combining capability and (ii) interference suppression capability. While interference suppression is the inherent capability of adaptive array antenna, multipath combining capability is gained thanks to the use of subband signal processing. We note here again that a conventional adaptive array performs only spatial processing (narrow-band beamforming), and thus does not have capability to combine multipath components. SBAA, on the other hand, was shown in [11] to be able to increase the multipath correlation, and thus has the capability to combine multipath components. We also compare the performance of the implicit 2D RAKE with that of the standard 2D RAKE to support our discussion in Section 2. The simulation model is given in Table 1.

For simplicity, when performing the simulation, we assume perfect synchronisation of the pilot signal and we use the recalculation method to obtain the output SINR. The 1000 data symbols are first used as the training symbols to

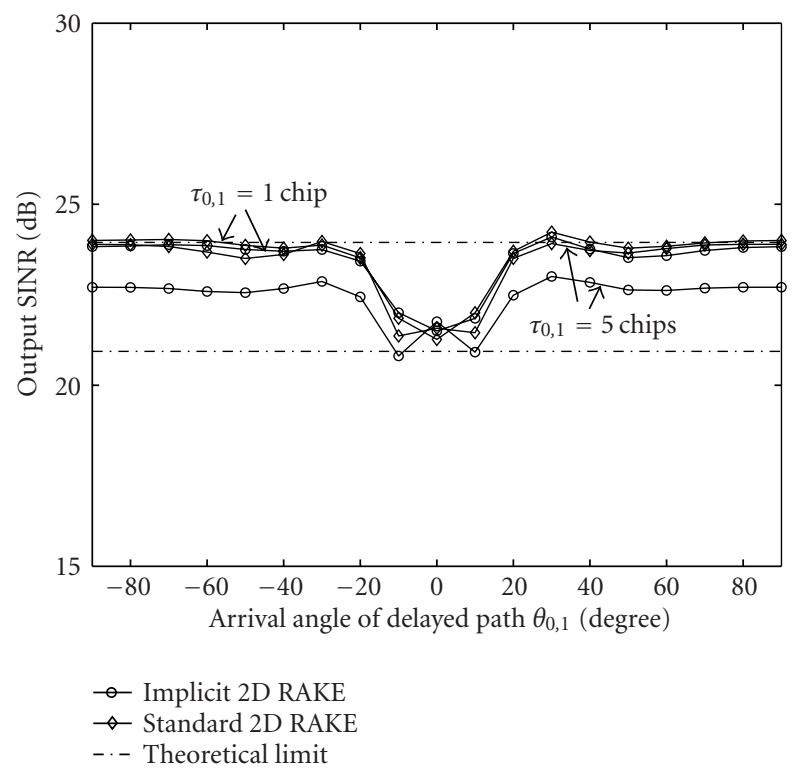

FIGURE 4: SINR versus AOA of delayed path $\left(\theta_{0,0}=0^{\circ} / \tau_{0,0}=0\right.$ chip, $\tau_{0,1}=1$ and 5 chips).

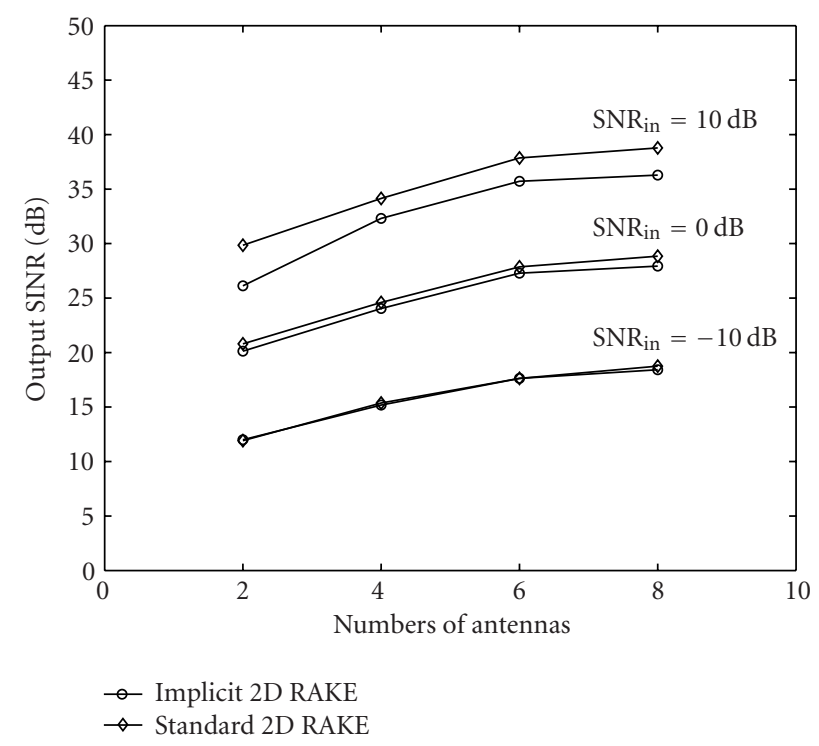

FIGURE 5: SINR versus number of antennas $\left(\theta_{0,0}=0^{\circ} / \tau_{0,0}=0\right.$ chip, $\theta_{0,1}=15^{\circ} / \tau_{0,1}=1$ chip, and $\theta_{0,2}=-20^{\circ} / \tau_{0,2}=2$ chips $)$.

obtain the optimal weights by SMI algorithm. These symbols are then used again as the data symbols to calculate the output SINR.

\subsection{Multipath combining capability}

The multipath combining capability of the implicit 2D RAKE is illustrated in Figures 3, 4, and 5. In Figure 3, we assume that there are two multipaths incident at the array: the direct path with $\theta_{0,0}=0^{\circ}$ and delay $\tau_{0,0}=0 \mathrm{chip}$, and the delayed path with $\theta_{0,1}=30^{\circ}$ and delay $\tau_{0,1}$ varying from 0 to 
TABLE 1

\begin{tabular}{l|l}
\hline \multicolumn{2}{c}{ Simulation model } \\
\hline $\begin{array}{l}\text { Type of array } \\
\text { Number of antennas }\end{array}$ & $\begin{array}{l}\text { Linear with } d=\lambda / 2 \\
\text { Number of subbands (number } \\
\text { of TDLs for standard 2D RAKE) }\end{array}$ \\
Type of modulation & $K=31$ \\
Data length & Direct-sequence BPSK \\
Spreading code & 1000 symbols \\
Adaptive algorithm & Gold code with $P_{G}=31$ \\
Input SNR & Sample matrix inversion \\
\hline
\end{tabular}

20 chips. It is noticed that the output SINR of the implicit 2D RAKE decreases gradually between the two theoretical limits as the delay of the delayed path increases. The upper limit is the SINR value when the two paths are completely correlated and are calculated using (14), while the lower limit is the SINR value calculated using (13) corresponding to the case in which the two paths are totally uncorrelated. It is also noted that the performance of the standard 2D RAKE is better than that of the implicit 2D RAKE in that the output SINR of the standard 2D RAKE is kept almost constant at the upper theoretical limit. The reason why the implicit 2D RAKE cannot achieve the same output SINR of the standard 2D RAKE is explained as follows. Since the standard 2D RAKE utilises TDLs to combine multipaths if the delay of multipaths are within the length of TDLs, the correlation between multipath components are fully maintained and thus its output SINR is maximised. On the other hand, the correlation between multipaths in each subband of the implicit 2D RAKE decreases as the delay of delayed paths increases [11], causing the output SINR to deteriorate as shown Figure 3. Thus it is clear that if the delay is smaller than $K$, the standard 2D RAKE combines multipaths better than the implicit 2D RAKE does. However, the practical DS-CDMA systems often suffer multipaths with delays of about several chips, and in such case, the implicit 2D RAKE can achieve relatively equivalent performance of the standard 2D RAKE particularly at low input SNR.

Figure 4 shows the output SINRs as the AOA of the delayed ray $\theta_{0,1}$ varies. It can be seen that if the delayed ray arrives at the array from an AOA significantly different from the direct ray, then better output SINR can be achieved by both the 2D RAKEs. The reason for this is that when the difference in the AOAs of the two paths is large enough, the 2D RAKEs can produce a supplementary lobe with a certain gain pointing towards the AOA of the delayed ray. By doing so, the power of the delayed ray is optimally combined to maximise the output SINR. Whereas when the difference in the AOAs is small, the 2D RAKEs cannot create the additional lobe, causing the two paths to share the same main lobe, and thus the power of the delayed path cannot be optimally combined, leading to poorer output SINR. It is also noticed that when the delay of the delayed path is small, namely, when $\tau_{0,1}=1$ chip, the performances of the implicit 2D RAKE and the standard 2D RAKE are almost the same. However, as the delay of the delayed path increases, the performance of the implicit 2D RAKE becomes worse than that of the standard 2D RAKE. For $\tau_{0,1}=5$ chips, the standard 2D RAKE can achieve approximately $1.7 \mathrm{~dB}$ better output SINR than the implicit 2D RAKE does.

Figure 5 compares the performances of the two 2D RAKEs for different number of antenna elements and input SNRs. In this case, we assume that the received signal contains three multipaths: the direct path with $\theta_{0,0}=0^{\circ} / \tau_{0,0}=0$ chip, the first delayed path with $\theta_{0,1}=15^{\circ} / \tau_{0,1}=1 \mathrm{chip}$, and the second delayed path with $\theta_{0,2}=-20^{\circ} / \tau_{0,2}=2$ chips. We define the input SNR as the power ratio of each path to the noise, and compare the performances of the two $2 \mathrm{D}$ RAKEs for 3 values of input SNR: $-10 \mathrm{~dB}, 0 \mathrm{~dB}$, and $10 \mathrm{~dB}$. It is seen from Figure 5 that the performances of the two 2D RAKEs are relatively equivalent, particularly, for low input SNRs. The reason why the implicit 2D RAKE cannot obtain the same output SINR as the standard 2D RAKE does at high input SNRs can be explained as follows. Since the signal power at the array output includes both the power of the desired signal and an amount of desired signal power correlated in the multipaths, the difference between output SINRs of the two 2D RAKE schemes depends mainly on the capability to extract the correlated signal power from multipaths. At low input SNR, since noise power is dominant, the output SINRs of both the two schemes thus are similar. However, at higher input SNR, the signal and the correlated signal power become dominant. Since the standard 2D RAKE has been shown to combine multipaths better, the correlated power it can extract from multipaths is thus larger than that the implicit 2D RAKE can do. Consequently, the SINR performance of the standard 2D RAKE is better than that of the implicit 2D RAKE at high input SNR.

\subsection{Interference suppression capability}

We now compare the MAI cancellation capabilities of the implicit 2D RAKE and the standard 2D RAKE. The propagation model is set up with one desired user and three other undesired users with interference to noise ratio INR $=0 \mathrm{~dB}$ as MAI sources. For each user's signal, we assume there are one direct ray and two delayed rays with AOAs and delays as given in Figure 6. In the figure, the denotation $a^{\circ} / d$ means that the path is incident at the array from arrival angle $a^{\circ}$ with $d$ chip delay. When there are no multipaths in all user's signals, that is, each user's signal contains only the direct path (with 0 delay), the propagation environment is called the interference only; whereas if there are multipaths, it is defined as the interference plus multipath environment.

The interference suppression capability of the two 2D RAKE schemes is shown in Figure 7, where the solid and the dotted lines denote the output SINRs of the interference plus multipath and the interference only environments, respectively. It is noticed that in the interference only environment, both the two 2D RAKEs have the same interference suppression capability. However, when there exist multipaths, the performance of the implicit 2D RAKE deteriorates about $1.5 \mathrm{~dB}$ compared with that of the standard 2D RAKE. Therefore, it is concluded that although the implicit 2D RAKE achieves the same interference suppression capability with 

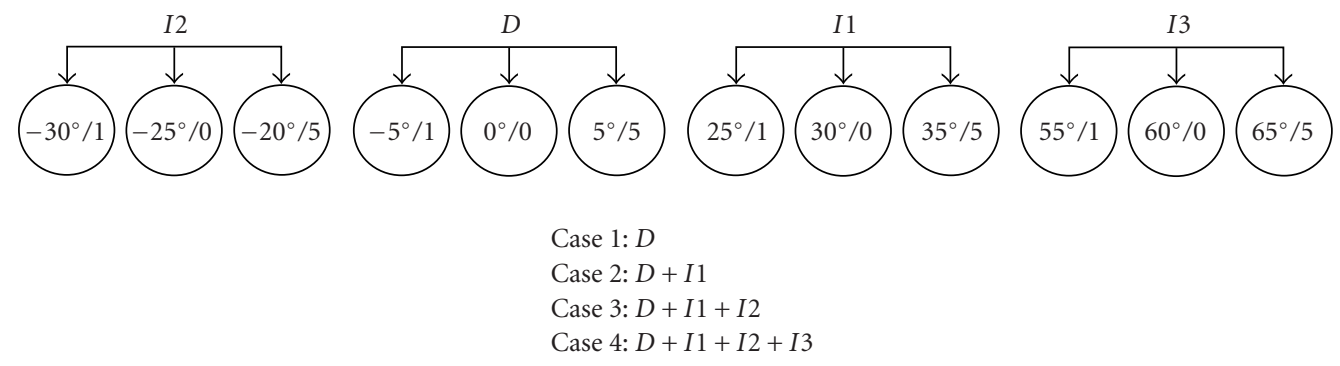

Figure 6: Propagation model with MAI interferences.

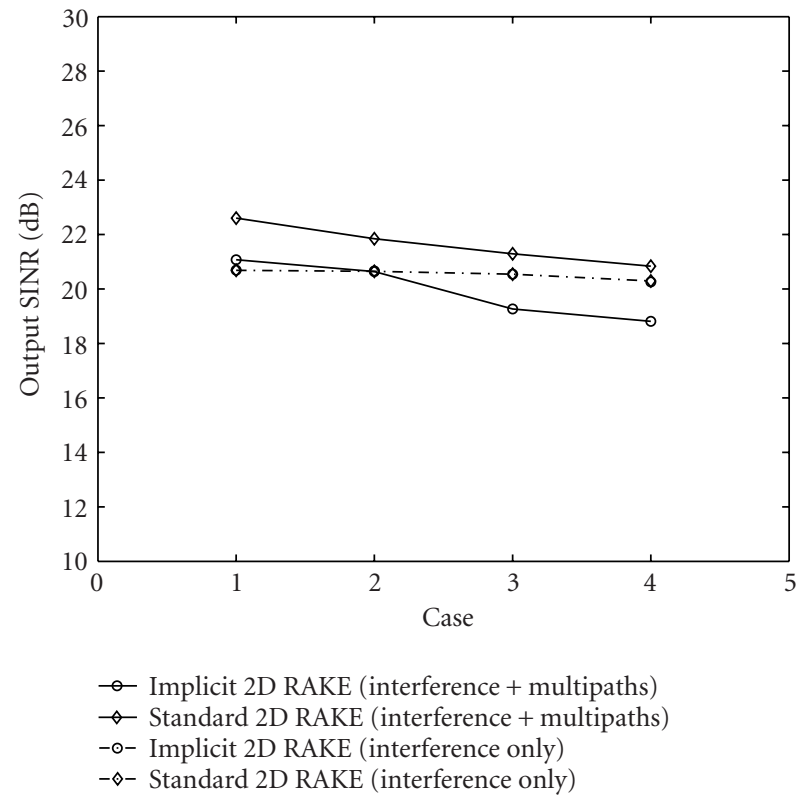

FIgURE 7: MAI cancellation capabilities.

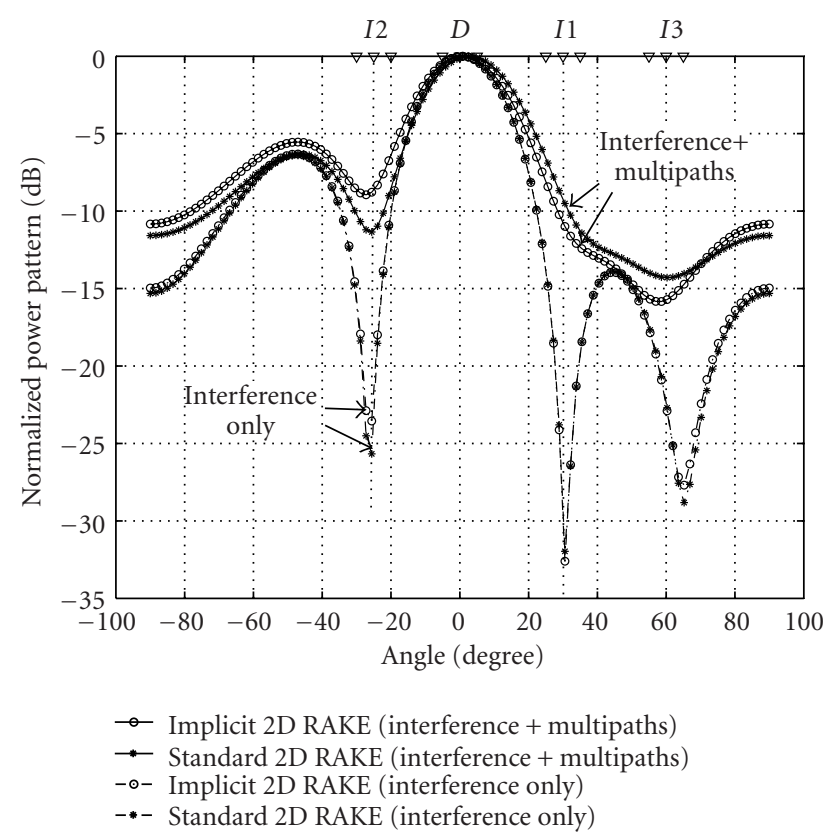

FIgURE 8: Normalised power patterns (Case 4 of Figure 6). that of the standard 2D RAKE, it suffers the problem of multipaths of the interferences more seriously than the standard 2D RAKE does.

The normalised power patterns of the two 2D RAKEs corresponding to Case 4 of Figure 6 are compared in Figure 8. It is observed that the two 2D RAKEs produce the same power patterns in the interference only environment. However, when there are multipaths, the power pattern of the implicit 2D RAKE becomes worse in that its nulls are still not correctly pointed toward the direct path of interferences causing the poorer performance. Note that the multipaths are not perfectly combined in the implicit 2D RAKE, particularly, with 32 subbands as used in the simulation. Therefore, the nulls are slightly inclined from the direction of interferences. For larger number of subbands or spreading code length, it is expected that the power patterns of the two $2 \mathrm{D}$ RAKE will be the same.

\section{CONCLUSION}

We have presented a novel configuration of subband adaptive array for DS-CDMA mobile radio which is called the implicit 2D RAKE. It is clearly shown that the implicit 2D RAKE can obtain relatively equivalent performance as the standard 2D RAKE does while saving a large amount of computational load. The proposed configuration therefore can be well applied for DS-CDMA systems to maximise the performance benefits.

It should be noted that the performance of the implicit can be improved to be the same with that of the conventional 2D RAKE by combining with cyclic prefix data transmission scheme [16]. For CDMA, we have introduced the so-called cyclic prefix spread code [15] which can maximise the diversity gain of the implicit 2D RAKE in multipath fading environment. This proposed scheme will be a topic in a different paper.

\section{REFERENCES}

[1] G. L. Turin, "The effect of multipath and fading on the performance of direct-sequence CDMA systems," IEEE Journal on Selected Areas in Communications, vol. 2, no. 4, pp. 597603, 1984.

[2] Y. Ogawa and T. Ohgane, "Advances in adaptive antenna technologies in Japan," IEICE Trans. Communications, vol. E84-B, no. 7, pp. 1704-1712, 2001. 
[3] R. T. Compton Jr, "An adaptive array in a spread-spectrum communications system," Proceedings of the IEEE, vol. 66, no. 3, pp. 289-298, 1978.

[4] R. Kohno, H. Imai, M. Hatori, and S. Pasupathy, "Combination of an adaptive array antenna and a canceller of interference for direct-sequence spread-spectrum multiple-access system," IEEE Journal on Selected Areas in Communications, vol. 8, no. 4, pp. 675-682, 1990.

[5] T. Inoue and Y. Karasawa, "Two-dimensional RAKE reception scheme for DS/CDMA systems in beam space digital beam forming antenna configuration," IEICE Trans. Communications, vol. E81-B, no. 7, pp. 1374-1383, 1998.

[6] B. H. Khalaj, A. J. Paulraj, and T. Kailath, "2D RAKE receivers for CDMA cellular systems," in IEEE. Global Telecommunications Conference (GLOBECOM'94), pp. 400-404, San Francisco, Calif, USA, December 1994.

[7] A. Stéphenne and B. Champagne, "Improving the performance of blind CDMA 2D RAKE receivers with phase ambiguity in the bit decision variable," in Proc. 32th Asilomar Conference on Signals, Systems and Computers, pp. 1882-1886, Pacific Grove, Calif, USA, November 1998.

[8] A. J. Paulraj and C. B. Papadias, "Space-time processing for wireless communications," IEEE Signal Processing Magazine, vol. 14, no. 6, pp. 49-83, 1997.

[9] H. Iwai, T. Shiokawa, and Y. Karasawa, "An investigation of space-path hybrid diversity scheme for base station reception in CDMA mobile radio," IEEE Journal on Selected Areas in Communications, vol. 12, no. 5, pp. 962-969, 1994.

[10] J. M. Khalab and M. K. Ibrahim, "Novel multirate adaptive beamforming technique," Electronics Letters, vol. 30, no. 15, pp. 1194-1195, 1994.

[11] Y. Zhang, K. Yang, and M. G. Amin, "Adaptive array processing for multipath fading mitigation via exploitation of filter banks," IEEE Trans. Antennas and Propagation, vol. 49, no. 4, pp. 505-516, 2001.

[12] X. N. Tran, T. Taniguchi, and Y. Karasawa, "Performance analysis of subband adaptive array in multipath fading environment," IEICE Trans. Fundamentals, vol. E85-A, no. 8, pp. 1798-1806, 2002.

[13] R. T. Compton Jr, "The relationship between tapped delayline and FFT processing in adaptive arrays," IEEE Trans. Antennas and Propagation, vol. 36, no. 1, pp. 15-26, 1988.

[14] X. N. Tran, T. Taniguchi, and Y. Karasawa, "Theoretical analysis of subband adaptive array combining cyclic prefix data transmission scheme," IEICE Trans. Communications, vol. E85-B, no. 12, pp. 2610-2621, 2002.

[15] T. Omata and Y. Karasawa, "Implicit 2D-RAKE function of subband signal processing adaptive array for spread spectrum systems with spreading code adding a cyclic prefix," Tech. Rep. AP2001-15, IEICE, May 2001.

[16] X. N. Tran, T. Taniguchi, and Y. Karasawa, "Subband adaptive array for multirate multicode DS-CDMA systems," IEICE Trans. Fundamentals, vol. E86-A, no. 7, pp. 1611-1618, 2003.

Xuan Nam Tran was born in Thanh Hoa, Vietnam, in 1971. He received his B.E. degree from Hanoi University of Technology, Vietnam, in 1993, M.E. degree in telecommunications engineering from the University of Technology, Sydney, Australia, in 1998, and Dr. Eng. degree from the University of Electro-Communications, Tokyo, Japan, in 2003. He is currently a Research Associate at the Department of Information Communications Engineering, The University of
Electro-Communications, Tokyo, Japan. His research interests are in the areas of space-time processing, space-time coding, and multiple input multiple output wireless communications systems. Dr. Tran is a member of the IEEE and IEICE, Japan.

Takanori Omata received the B.S. degree in electrical engineering from the University of Electro-Communication in 2001. He joined the Department of Core Network Planning, J-Phone East Co., Ltd. (Vodafone K.K.) in 2001, where he has involved in designing core network.

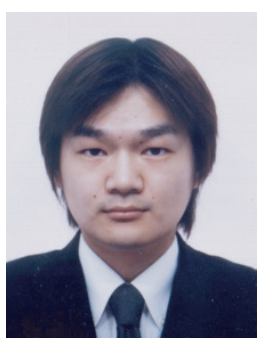

Tetsuki Taniguchi received the B.S. and M.S. degrees in electrical engineering from Tokyo Metropolitan University, Tokyo, Japan, and D.E. degree in natural science from Kanazawa University, Kanazawa, Japan, in 1989, 1991, and 1996, respectively. In 1992, he joined Kanazawa University, where he worked as a Research Assistant at the Department of Electrical and Information Engineering, and a Researcher at the

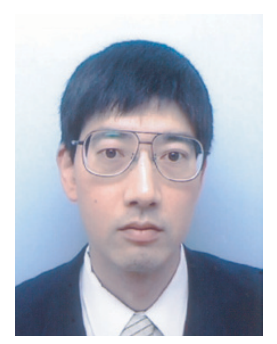
Laboratory of Magnetic Field Control and Applications. In 2001, he joined The University of Electro-Communications, where he is currently a Research Assistant at the Department of Electronic Engineering. His research interests are in digital signal processing, digital communications, and nondestructive evaluation. $\mathrm{He}$ is a member of the IEEE, the Institute of Electrical Engineers of Japan, and the Japan Society of Applied Electromagnetics and Mechanics.

Yoshio Karasawa received his B.E. degree from Yamanashi University in 1973 and M.S. and Dr. Eng. degrees from Kyoto University in 1977 and 1992, respectively. He joined KDD R\&D Labs. in 1977. From July 1993 to July 1997, he was a Department Head of ATR Optical and Radio Communications Research Laboratories and ATR Adaptive Communications Research Laboratories, both in Kyoto. From 1997 to 1999,

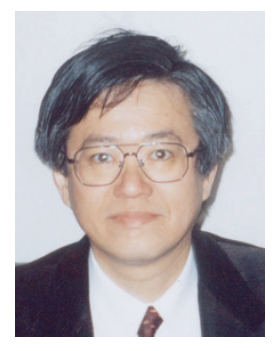
he was a Senior Project Manager of KDD R\&D Labs. Currently, he is a Professor at The University of Electro-Communications, Tokyo. Since 1977, he has been engaged in studies on wave propagation and radio communication antennas, particularly on theoretical analysis and measurements for wave-propagation phenomena, such as multipath fading in mobile radio systems, tropospheric and ionospheric scintillation, and rain attenuation. His recent interests are in frontier regions bridging "wave propagation" and "digital transmission characteristics" in wideband mobile radio systems and digital signal processing antennas. Dr. Karasawa received the Young Engineers Award from the Institute of Electronics and Communication Engineers (IECE) of Japan in 1983 and the Meritorious Award on Radio from the Association of Radio Industries and Businesses (ARIB), Japan, in 1998. He is a member of the IEEE and URSI. 